\title{
JOINT VECTOR MAGNETOGRAPH OBSERVATIONS AT BBSO, HUAIROU STATION AND MEES SOLAR OBSERVATORY
}

\author{
HAIMIN WANG, JOHN VARSIK, HAROLD ZIRIN
}

Big Bear Solar Observatory, California Institute of Technology, Pasadena, CA 91125, U.S.A.

RICHARD C. CANFIELD, K. D. LEKA

Mees Solar Observatory, University of Hawaii at Manoa, Honolulu, HI 96822, U.S.A.

and

JINGXIU WANG

Beijing Astronomical Observatory, Chinese Academy of Sciences, Beijing, China

(Received 13 May, 1991; in revised form 27 April, 1992)

\begin{abstract}
Joint vector magnetograph observations were carried out at Big Bear Solar Observatory (BBSO), Huairou Solar Observing Station (Huairou), and Mees Solar Observatory (MSO) in late September 1989. Comparisons of vector magnetograms obtained at the three stations show a high degree of consistency in the morphology of both longitudinal and transverse fields. Quantitative comparisons show the presence of noise, cross-talk between longitudinal field and transverse field, Faraday rotation and signal saturation effects in the magnetograms. We have tried to establish how the scatter in measurements from different instruments is apportioned between these sources of error.
\end{abstract}

\section{Introduction}

Observations of solar vector magnetic fields are of vital importance in understanding solar activity. Hagyard and her colleagues at Marshall Space Flight Center (MSFC) have led the way in the measurements of vector magnetic fields, the interpretation of the data, and the study of the relationship between vector magnetic fields and solar flares (Hagyard, 1988; Hagyard and Rabin, 1986; Hagyard et al., 1982, 1984; Hagyard, Gary, and West, 1988). Many other solar observatories have recently made a great effort to establish vector magnetograph systems with high accuracy and high temporal and spatial resolution. Ronan et al. (1992) compared vector magnetogram measurements of MSFC and MSO, and showed a number of techniques to evaluate the operation of the two very different systems. In this paper, we compare the vector magnetograms obtained at the Big Bear Solar Observatory, the Huairou Solar Observing Station of Beijing Astronomical Observatory in China, and the Mees Solar Observatory of the University of Hawaii, to determine how well the measurements of vector fields at the three observatories agree, and to evaluate how the scatter between different instruments is apportioned between various sources of error.

\section{Instruments}

The videomagnetograph (VMG) system at BBSO was developed by Leighton and Smithson (Mosher, 1976). Its recent development was described by Zirin (1985). The

(C) 1992 Kluwer Academic Publishers. Printed in Belgium. 
system has made valuable longitudinal field observations for almost two decades. BBSO started to experiment with vector field measurement in the summer of 1988. In the first paper on this subject, Cacciani, Varsik, and Zirin (1989) described the BBSO system for vector field measurements using a magneto-optical filter (MOF). In the observation presented in this paper, the setup of the system is almost the same as that described by Cacciani, Varsik, and Zirin, except that a $\frac{1}{4} \AA$ Zeiss birefringent filter was used instead of the MOF. The current system uses a narrow photospheric spectrum line, Ca II $6103 \AA$ line $\left(g_{L}=2.0\right)$. The center of the bandpass is set at $\Delta \lambda=66 \mathrm{~m} \AA$ on the blue side of the spectral line. The temporal and spatial resolution of $\mathrm{BBSO}$ vector magnetograms depends on the number of individual frames that are added to produce them; the magnetograms used in this paper are made from 128 video frames for longitudinal field, 144 for transverse field; they have a time resolution of about $1 \mathrm{~min}$ and a spatial resolution of 1-2 arc sec.

The VGM system at the Huairou Station was developed by Ai (1987). The BBSO and Huairou systems are very similar, except that Huairou uses the Fer $5324 \AA$ line $\left(g_{L}=1.5\right)$, and sets the filter at $\Delta \lambda=75 \mathrm{~m} \AA$ from line center. Like the BBSO magnetograms, the temporal and spatial resolution of its magnetograms depends on the number of video frames that are added. The Huairou magnetogram used in this paper is the sum of 256 individual video frames for both longitudinal and transverse fields, corresponding to a temporal resolution of $5 \mathrm{~min}$ and a spatial resolution of $2 \mathrm{arc} \mathrm{sec}$.

At BBSO and Huairou, each set of observing data consists of narrow-band images (filtergrams) in each of the four Stokes parameters: $I$, the direct intensity image; $V$, the difference between left and right circularly-polarized images; $U$, the difference between two orthogonal linearly-polarized images in a certain azimuthal direction; $Q$, the same as $U$, but with a $45 \mathrm{deg}$ change in the azimuthal angle. $V$ provides the signal for the longitudinal field, $Q$ and $U$ for the transverse field. The amplitude of the transverse field is determined by

$$
B_{T}=C\left(Q^{2}+U^{2}\right)^{1 / 4},
$$

where $C$ is a constant, and the azimuthal angle is determined by

$$
\theta=\frac{1}{2} \arctan \left(\frac{U}{Q}\right)
$$

The Stokes's polarimeter at MSO (Mickey, 1985) uses a much different observational and analytical approach. It uses a spectrograph to observe $I, Q, U$, and $V$ line profiles in 128 different wavelength channels (each about $25 \mathrm{~m} \AA$ wide) at only a single point on the Sun, in contrast to four narrow-band Stokes images centered on only a single spectral band. Because it observes only one spatial point at a time, that point (a $6^{\prime \prime}$ aperture) must be scanned across the Sun's image to produce a solar image. Hence, it has much lower temporal resolution than the BBSO or Huairou instruments (about 1 hour). When sampled each 5.6", as in this paper, this instrument produces magnetograms that have much lower spatial resolution (about $6^{\prime \prime}$ ) than those from BBSO or 
Huairou. On the other hand, the more complete spectroscopic nature of the data allows a more sophisticated analysis. For low total polarization (less than $1 \%$ ) the magnetic field values are derived using the method of Ronan, Mickey, and Orrall (1987), which is based on the same weak-field approximation as Equations (1) and (2). For larger total polarization the nonlinear, least-squares Unno-fitting code of Skumanich and Lites (1987) is used. The MSO magnetogram used in this paper is derived by applying the Unno code simultaneously to the profiles of the two main spectral lines observed ( $\mathrm{Fe}$ I 6301.5 and $6302.5 \AA$ ). The analysis takes into account magneto-optic and scattered light effects, including unresolved, unmagnetized regions.

The longitudinal magnetograms are calibrated at all three observatories. Only MSO provides a calibration for transverse fields, so we calibrated the BBSO and Huairou transverse field values with the MSO transverse magnetogram by linear regression.

\section{Morphological Comparisons}

At the end of September 1989, active region No. 1761 (NOAA No. 5702) was followed by all three observatories. We do not have a single data set containing simultaneous observations at all three stations. However, it is straightforward to compare nearly simultaneous data of BBSO vs MSO and BBSO vs Huairou, respectively. Figures 1 and 2 compare such pairs of magnetograms. The preliminary results of the comparisons have been reported at the NSO Solar Polarimetry Workshop (Wang, 1990). Since the workshop, the data reduction procedure of MSO has been improved and a better calibration was used in the inversion program of MSO. As a result, the MSO magnetogram in Figure 1 has been modified slightly.

Figure 1 compares magnetograms obtained by BBSO at 19:55 UT, September 22, and by MSO scanned from 18:21 to 19:31 UT, September 22. Figure 2 compares vector magnetograms obtained by BBSO at $00: 20 \mathrm{UT}$, and by Huairou at $00: 30 \mathrm{UT}$, September 24, 1989. We have rebinned the BBSO and Huairou magnetogram to match the coarser pixel spacing of MSO, which is $5.6 \mathrm{arc} \mathrm{sec}$. Careful visual comparison between the two plots of Figures 1 and 2 show a high similarity of measurements by the three stations, for both longitudinal and transverse fields.

Before any vector magnetograph was in operation, $\mathbf{H} \alpha$ observation was used as a unique tool to study the direction of magnetic field lines (Zirin, 1972). The dark fibrils in $\mathrm{H} \alpha$ mark lines of force connecting opposite magnetic polarity. Wang (1990) has shown that for the BBSO data used in this paper, the orientation of dark fibrils is generally consistent with the direction of the transverse fields. He also showed that the transverse fields of naked sunspots observed near the solar disk center have clear radial structures, so do the $\mathrm{H} \alpha$ fibril structures of those spots.

\section{Quantitative Comparison}

In order to make a more quantitative comparison between pairs of magnetograms obtained independently at different observatories, we have co-registered such pairs and 

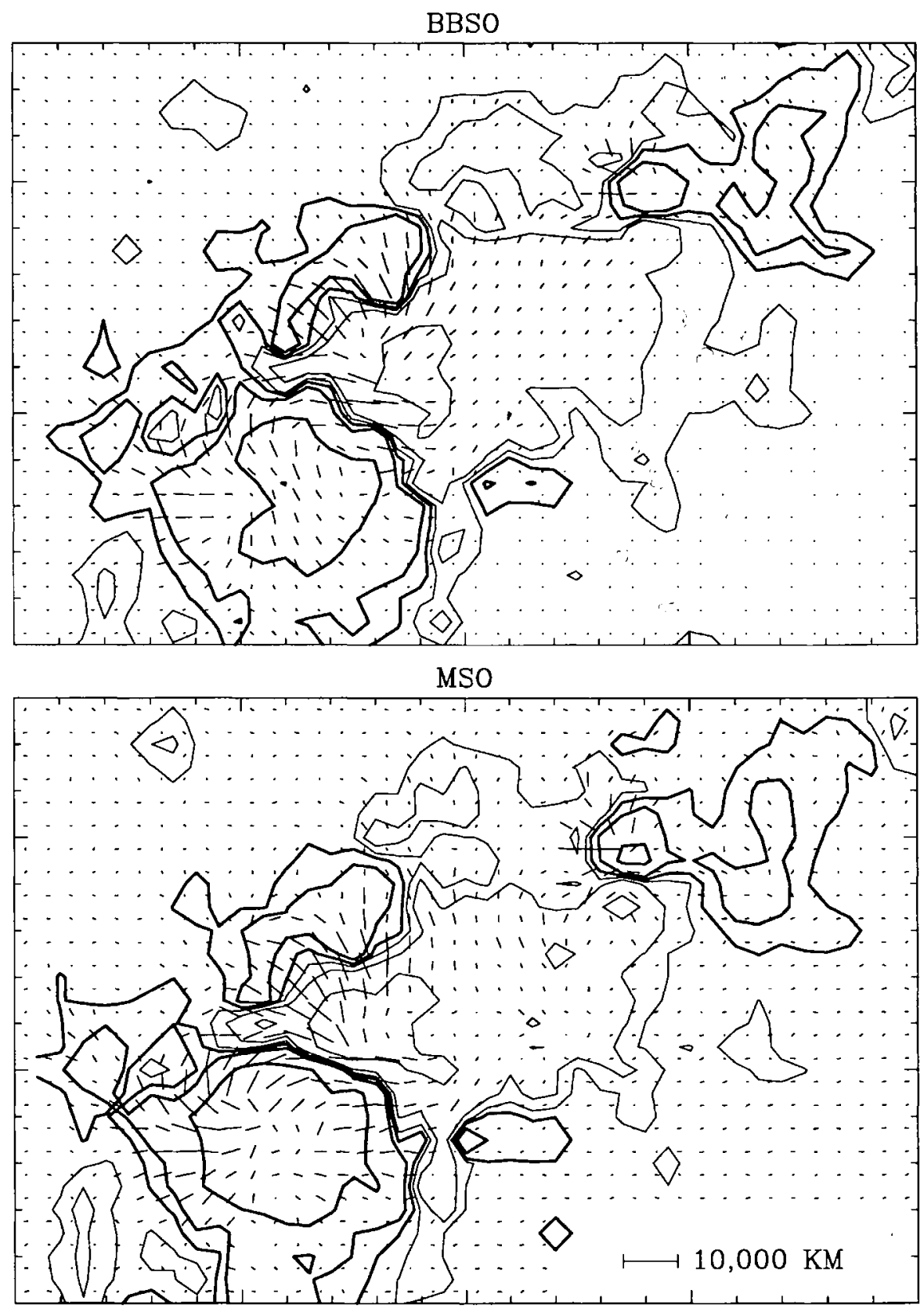

Fig. 1. Comparison between a pair of vector magnetograms obtained at BBSO and MSO, at about 19:30 UT, September 22, 1989. The longitudinal fields are presented by contours: darker lines indicate positive fields, lighter lines, negative fields. Four contour levels are plotted for each polarity: 100, 400, 1600, $3200 \mathrm{G}$. Transverse fields are plotted with bars. The length of the bars is proportional to the transverse field strength. The longest bars in each magnetogram are roughly $1500 \mathrm{G}$.

interpolated them onto identical grids. To minimize systematic distortions in the transverse field values, we have done this interpolation with polarization values, not field values.

The quantitative comparison between the BBSO and MSO magnetograms is shown in Figure 3. (a) Scatter plot of longitudinal fields; (b) scatter plot of transverse field strength; (c) difference of azimuthal angle as a function of MSO's transverse field strength; and (d) scatter plot of azimuthal angle for pixels with transverse field strength 

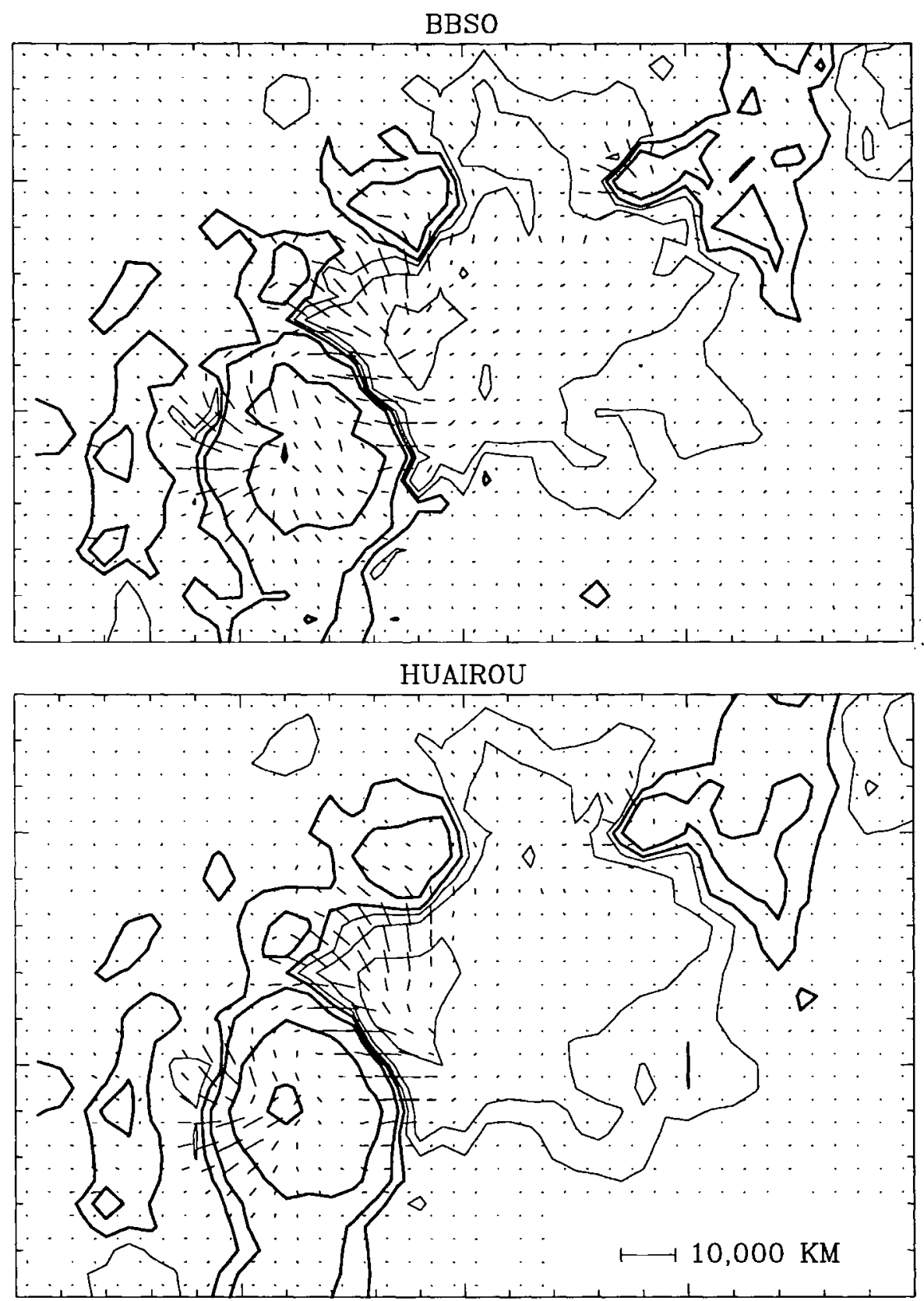

Fig. 2. Comparison between a pair of vector magnetograms obtained at BBSO and Huairou Station, at about 00:30 UT, September 24, 1989.

larger than $1200 \mathrm{G}$. Figure 4 repeats the same comparisons for BBSO and Huairou magnetograms.

In Table I we listed the scatter of measured quantities as a function of field strength. The scatter is defined as the root-mean-square deviation of measured quantities between two instruments. For the comparison of $B_{L}$, the field strength in the first column of the table represents longitudinal field strength, for the comparison of $B_{T}$ and azimuth, it represents transverse field strength.

Obviously, the measurements of BBSO and Huairou agree better than those of BBSO and MSO, and the measurements of longitudinal fields agree better than those of 

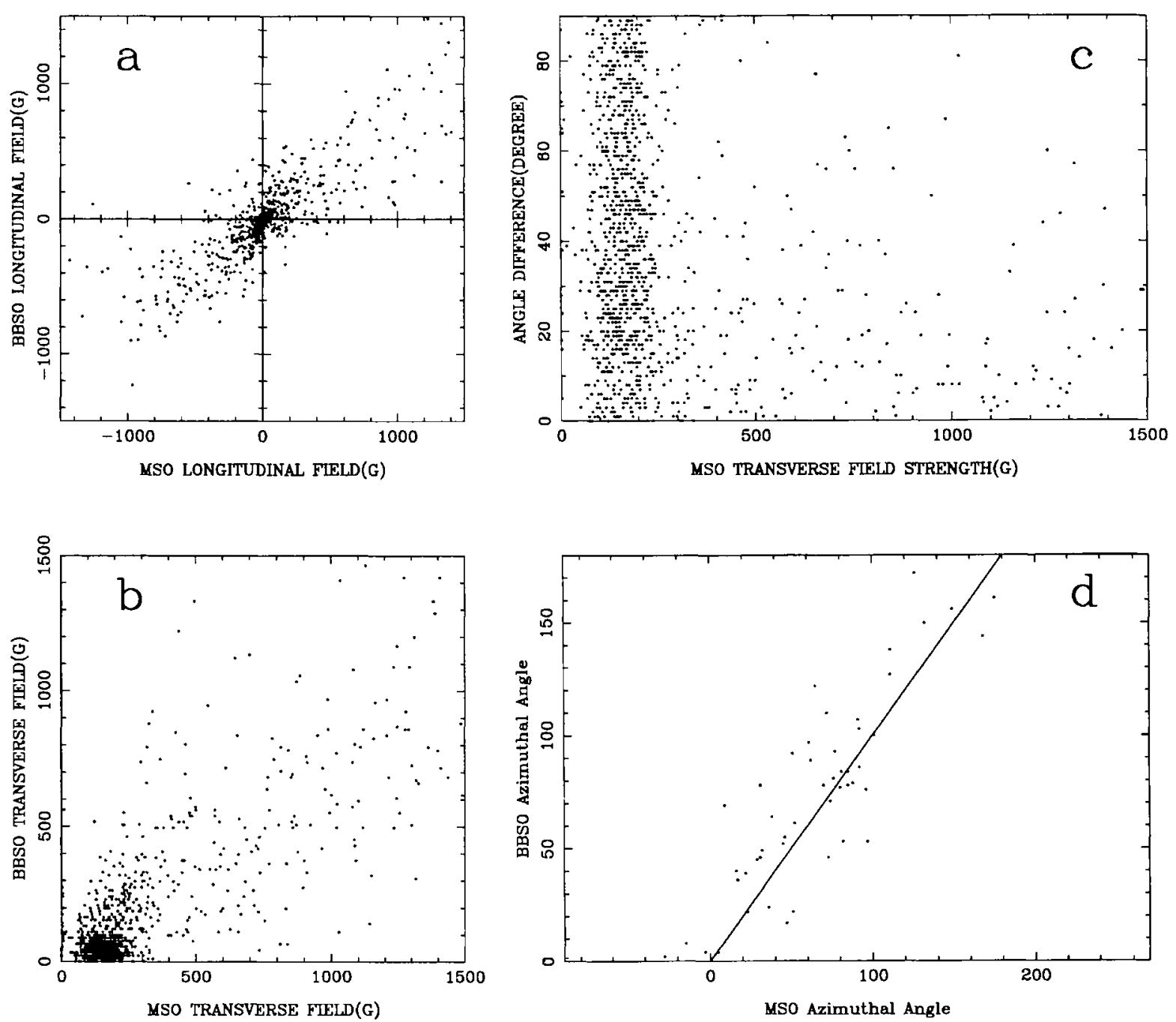

Fig. 3. Comparison of individual points in the BBSO and MSO magnetograms. (a) Line-of-sight field; (b) transverse field amplitude; (c) transverse field azimuth difference as a function of transverse field amplitude; (d) scatter plot of azimuthal angle for the points having transverse field stronger than $1200 \mathrm{G}$.

transverse fields. The possible sources of scatter include saturation effects at BBSO, cross talk between longitudinal fields and transverse fields, Faraday rotation, the lower spatial resolution at MSO, the inability to co-register the two magnetograms perfectly, and the lack of exact simultaneity of the two magnetograms. It is not easy to establish how the scatter is apportioned between all the sources of error, but we would like to give the following quantitative analyses:

\section{(1) Saturation Effect}

BBSO uses a single fixed position of the filter bandpass relative to line center (weak field approximation). MSO uses a spectrograph to observe full-line profiles in 128 different wavelength channels. Also during the conversion process, MSO takes the filling factor into account, BBSO does not. So a saturation effect would be expected for strong fields at BBSO. The saturation appears clearly in Figure 3(a), where most of the BBSO field 

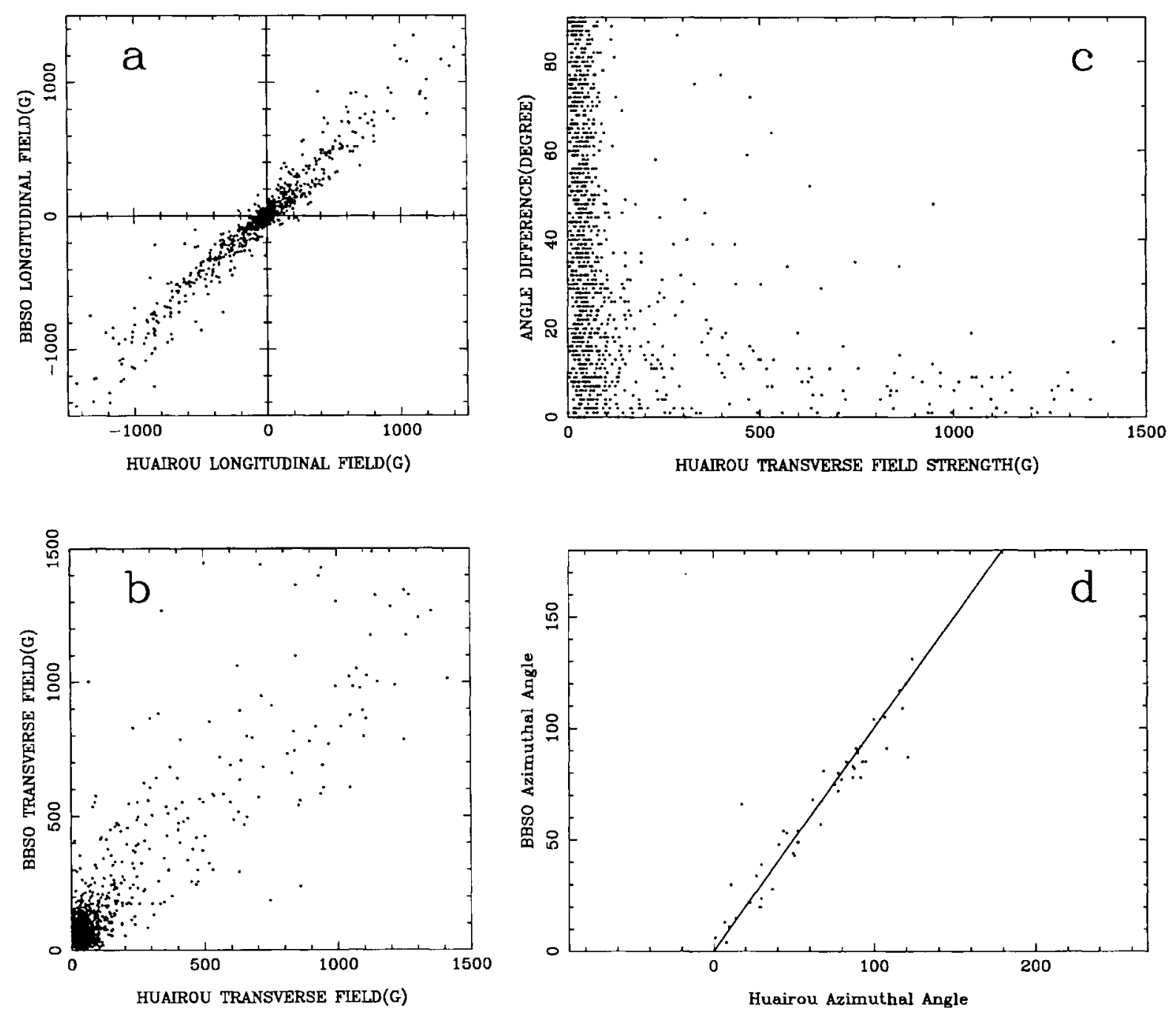

Fig. 4. Comparison of individual points in the BBSO and Huairou magnetograms. (a) Line-of-sight field; (b) transverse field amplitude; (c) transverse field azimuth difference as a function of transverse field amplitude; (d) scatter plot of azimuthal angle for the points having transverse field stronger than $1200 \mathrm{G}$.

TABLE I

Scatter in the measured quantities

\begin{tabular}{|c|c|c|c|c|c|c|}
\hline \multirow{2}{*}{$\begin{array}{l}\text { Field strength } \\
\text { (G) }\end{array}$} & \multicolumn{3}{|c|}{ BBSO vs MSO } & \multicolumn{3}{|c|}{ BBSO vs Huairou } \\
\hline & $B_{L}$ & $B_{T}$ & Azimuth & $B_{L}$ & $B_{T}$ & Azimuth \\
\hline $0-300$ & $63 \%$ & $75 \%$ & $41^{\circ}$ & $39 \%$ & $57 \%$ & $39^{\circ}$ \\
\hline $300-600$ & $58 \%$ & $60 \%$ & $25^{\circ}$ & $31 \%$ & $59 \%$ & $24^{\circ}$ \\
\hline $600-900$ & $40 \%$ & $55 \%$ & $22^{\circ}$ & $23 \%$ & $40 \%$ & $12^{\circ}$ \\
\hline $900-1200$ & $51 \%$ & $46 \%$ & $11^{\circ}$ & $17 \%$ & $30 \%$ & $7^{\circ}$ \\
\hline $1200-1500$ & $44 \%$ & $42 \%$ & $17^{\circ}$ & $20 \%$ & $18 \%$ & $6^{\circ}$ \\
\hline
\end{tabular}


strengths fall below those of MSO. For $B>1000 \mathrm{G}$, the saturation of longitudinal field contributes about $25 \%$ to the total scatter. This also explains the large increase of scatter between BBSO-MSO $B_{L}$ when $B_{L}>900 \mathrm{G}$. Saturation effects may also appear in the transverse field; however, since the MSO values are used to calibrate those of BBSO, it is not easy to separate the saturation effect and the inaccuracy in this kind of calibration method. The noise in the transverse field peaks around $180 \mathrm{G}$ in the MSO data and $60 \mathrm{G}$ in the BBSO data (Figure 3(b)). Both magnetograms are based on the weak field method in this range, since the polarization at most such points falls below the $1 \%$ level above which the MSO magnetograms are reduced using the Unno method, and we expect the transverse field to have a value simply related to the individual noise levels in Stokes $Q$ and $U$. The distribution of angle differences shows a random distribution at transverse field values less than about $250 \mathrm{G}$, which corresponds to the MSO noise level. Above that value the root mean square angle difference gradually decreases as the field strength increases (Figure 3(c)).

The quantitative comparison between BBSO and Huairou shows much less scatter, as one would expect from the almost identical nature of the two instruments and the small time difference between the two magnetograms. Figure 4(a) shows much less scatter than Figure 3(a), and shows no nonlinearity, implying that the two different magnetograms do not saturate at much different longitudinal field values. (One expects the weak field approximation to be valid for the $5324 \AA$ line at $\Delta \lambda=75 \mathrm{~m} \AA$ only for $B \ll 3800 \mathrm{G}$.) These magnetograms have been calibrated independently, using solar rotation; clearly the calibrations agree very well.

\section{(2) Cross-Talk}

The cross-talk at BBSO is caused by the leakage of circular polarization $V$ to linear polarization $Q$ and $U$. One possible cause is that when the temperature changes, the KDP does not remain at exactly $\frac{1}{4}$ wavelength. This effect causes about $10 \%$ of the error in the transverse field strength. The cross-talk also affects the azimuthal angle substantially, as is shown in the following analysis: if we plot the angle difference as a function of $B_{T}$, the difference decreases as $B_{T}$ increases (see Figures 3(c) and 4(c), and Table I); however, if we show them as a function of $B_{L}$, the scatter is distributed as follows:

$$
\begin{aligned}
B_{L} & =0-300 \mathrm{G}, & \Delta \theta & =40.5^{\circ}, \\
B_{L} & =300-600 \mathrm{G}, & \Delta \theta & =19.9^{\circ}, \\
B_{L} & =600-900 \mathrm{G}, & \Delta \theta & =19.2^{\circ}, \\
B_{L} & =900-1200 \mathrm{G}, & \Delta \theta & =29.3^{\circ}, \\
B_{L} & =1200-1500 \mathrm{G}, & \Delta \theta & =39.4^{\circ} .
\end{aligned}
$$

Obviously, when $B_{L}$ increases, it adds more contaminated signal to $Q$ and $U$, and $\Delta \theta$ increases. So transverse field data in umbrae have the largest cross-talk. The cross-talk effect of MSO is negligible $(<0.3 \%)$, and that of Huairou is about $2 \%$. 


\section{(3) Faraday Rotation}

Ronan et al. (1992) found a systematic offset (about $20^{\circ}$ ) in the azimuth relationship for the sunspot field in the MSFC-MSO comparison. They explained a large part of this offset by the Faraday rotation effect in the MSO magnetogram. We did the similar experiment for our comparisons. We include scatter plots of azimuthal angles for the pixels having transverse field strength above $1200 \mathrm{G}$ in Figures 3(d) and 4(d), for BBSO-MSO and BBSO-Huairou comparison, respectively. The BBSO-MSO comparison shows a systematic offset of about $10^{\circ}$, no offset appears in the BBSO-Huairou comparison. However, when we plot the scatter of azimuthal angle for field strength above $300 \mathrm{G}$, no offset is detectable; there is a weak signal of offset for field strengths above $600 \mathrm{G}$ in the BBSO-MSO comparison. The above symptoms may demonstrate that there is a Faraday effect contributing a systematic error up to $10^{\circ}$ in the BBSO-MSO comparison.

\section{(4) Misregistration, Lack of Exact Simultaneity, and Lower Spatial Resolution for MSO}

We estimated what fraction of scatter could be attributed to misregistration by the following test: we shifted the BBSO longitudinal magnetogram relative to the registered Huairou longitudinal magnetogram by 3 arc sec (about half of the MSO pixel size, it is the largest possible amount of misregistration that we would not notice), it caused about 10 to $15 \%$ of additional scatter in the field strength for all the field strength bins and $10 \mathrm{deg}$ in the azimuthal angles. The actual amount of error due to mis-registration is less than these values.

The error caused by lack of simultaneity was tested by comparing a pair of BBSO magnetograms about two hours apart. The scatters are about 3 to $5 \%$ for the longitudinal and transverse field strength, $3^{\circ}$ for the azimuthal angle.

Since we averaged BBSO polarization $(Q, U, V)$ measurements instead of the field strength to match the spatial resolution of MSO, we believe that the large difference in the spatial resolution does not contribute much to the total scatters. However, MSO takes account of the filling factor in the data inversion, BBSO does not. We do not know how to estimate the error caused by this difference.

Finally, we would like to discuss the inclination angle briefly. The accuracy of the inclination angle depends on that of both longitudinal and transverse field strengths. Since the BBSO and Huairou transvese fields do not have independent calibrations, there is no way to estimate the systematic error caused by the relative calibration of longitudinal and transverse fields. However, the random scatter of inclination angle can be estimated by using the random scatters of longitudinal and transverse field strength. E.g., when $B_{L}$ and $B_{T}$ are $1000 \mathrm{G}$, the error of inclination angle is about $19^{\circ}$ for the BBSO-MSO comparison, and $9^{\circ}$ for the BBSO-Huairou comparison. Smaller errors are expected for stronger fields; larger errors, for weaker fields. 


\section{Acknowledgements}

We are grateful to the staffs of all three observatories for support during the observations and Bruce Lites for making his Unno code available. We wish to thank the referee for helpful comments. The operation of the BBSO magnetograph and data reduction were supported by NASA under NAGW-1972 and by the NSF under ATM-8816007. Huairou Observing Station is supported by the Chinese National Science Foundation. The operation of the MSO Stokes Polarimeter and data reduction were supported through NASA grant NAGW-1542, and through Lockheed under NASA Contract NAS8-37344 with the NASA Marshall Space Flight Center.

\section{References}

Ai, G.: 1987, Publ. Beijing Astron. Obs. 9, 27.

Cacciani, A., Varsik, J., and Zirin, H.: 1989, Solar Phys. 125, 173.

Hagyard, M. J.: 1988, Solar Phys. 115, 107.

Hagyard, M. J. and Rabin, D. M.: 1986, Adv. Space Rev. 6, 7.

Hagyard, M. J., Gary, G. A., and West, E. A.: 1988, NASA Technical Memorandum 4048.

Hagyard, M. J., Cumings, N. P., West, E. A., and Smith, J. E.: 1982, Solar Phys. 80, 33.

Hagyard, M. J., Smith, J. B., Jr., Teuber, D., and West, E. A.: 1984, Solar Phys. 91, 115.

Mickey, D. L.: 1985, Solar Phys. 97, 223.

Mosher, J.: 1976, BBSO preprint.

Ronan, R. S., Mickey, D. L., and Orrall, F. Q.: 1987, Solar Phys. 113, 353.

Ronan, R. S., Orrall, F. Q., Mickey, D. L., West, E. A., Hagyard, M. J., and Balasubramaniam, K. S.: 1992, Solar Phys. 138, 49.

Skumanich, A. and Lites, B. W.: 1987, Astrophys. J. 322, 473.

Wang, H.: 1990, in L. November (ed.), Solar Polarimetry, Proceedings of the Workshop of Solar Polarimetry,

National Solar Observatory, Sacramento Peak, NM.

Zirin, H.: 1972, Solar Phys. 22, 34.

Zirin, H.: 1985, Australian J. Phys. 38, 961. 